\title{
EXTRACT FROM PREFACE TO THE GERMAN EDITION
}

THE present book has been compiled from lectures which I delivered at the University of Vienna. The object of these lectures was to explain the fundamental ideas and the most important consequences of modern atomic theory in a clear and elementary fashion, avoiding the use of higher mathematics. Since only a "school" knowledge of physics is assumed on the part of the reader I hope that this book may be of use, not merely to physicists, but also to chemists and even, perhaps, to those who, without possessing any great knowledge of science, wish to learn something of atomic theory. . . .

Arthur HaAs. 
